\title{
Produktion von Dünnschichtsolarzellen
}

\author{
D. Bonnet, M. Harr'
}

\begin{abstract}
Auf Grund der hohen Leistungen von Kadmiumtellurid-Dünnschichtsolarzellen, die durch schnelle und unempfindliche Prozesse hergestellt werden, zeigt die Technologie ein großes Potential für die industrielle Produktion. ANTEC Solar GmbH hat mit Erfolg die finanzielle Basis geschaffen, um die Produktion in einem Maßstab von $10 \mathrm{MW}$ pro Jahr aufzunehmen. Hierbei wird der Prozeß der Close-Spaced-Sublimations für die Abscheidung der Halbleiterschichten benutzt. Grundlegende technische und kaufmännische Überlegungen werden präsentiert und die Fabrik im Bau wird erläutert.
\end{abstract}

\section{Schlüsselwörter: Solarenergie; Photovoltaik; Dünnschichtsolarzellen}

Production of thin-film solar cells. The high performance of CdTe thin-film solar cells made by fast and rugged manufacturing process indicates a significant promise for commercial production. ANTEC Solar GmbH has successfully created the financial basis for production on a scale of $10 \mathrm{MW}_{\mathrm{p}}$ per annum using close-spaced sublimation for the deposition of the semiconductor films. We present basic technical and business considerations comment the plant in construction.

Keywords: solar energy; photovoltaics; thin-film solar cells

\section{Einleitung}

Im Hinblick auf die kostengünstige Nutzung der Solarenergie haben Dünnschichtsolarzellen grundlegende Vorteile durch geringen Materialverbrauch, integrierte Modul-Definition und Massenproduktion. Sie können ein Kostenlimit von 0,6 EURO pro Watt bereits bei einer Kapazität von $60 \mathrm{MW}$ pro Jahr erreichen [1], während kristalline Siliziummodule dieses Kostenniveau erst bei Kapazitäten von erheblich mehr als $500 \mathrm{MW}$ pro Jahr erreichen [2]. Die Wahl des Materials und der Herstellungstechnik werden direkt den Vorteil des individuellen Systems bestimmen. ANTEC Solar hat Kadmiumtellurid als das meistversprechende Material und close-spaced-sublimation (CSS) als Abscheideprozeß gewählt, um die Massenproduktion aufzunehmen. Diese Entscheidung basierte auf aussichtsreichen Resultaten eigener Arbeiten seit 1991 und auf der Analyse vergangener industrieller Bemïhungen auf diesem Gebiet. Die wesentlichen Faktoren für eine erfolgreiche Produktion wurden identifiziert und bewertet. Im folgenden werden die Ergebnisse dieser Analysen diskutiert und nach einer kurzen Übersicht vergangener industrieller Bemühungen werden

\footnotetext{
Dr. Dieter Bonnet, leitender Wissenschaftler der ANTEC $\mathrm{GmbH}$, Industriestraße 2, D-65779 Kelkheim; Dr. Michael Harr, Geschäftsführer der ANTEC Solar GmbH, Arnstädter Straße 22, D-99334 Rudisleben.
}

das Konzept und der Status der Bemühungen zur Industrialisierung zusammen mit dem Plan für die Fabrik wiedergegeben.

\section{Dünnschichtsolarzellen in der Vergangenheit}

Über mehr als dreißig Jahre war das Geschäftspotential von Dünnschichtsolarzellen als kostengünstige effiziente Wandler von solarer Strahlung in Elektrizität die Ursache industrieller Bemühungen. Weniger als eine Handvoll von Halbleitern wurde identifiziert, die hierzu ausreichendes Potential boten. Um 1971 wurde die $\mathrm{Cu}_{2} \mathrm{~S} / \mathrm{CdS}$-Dünnschichtsolarzelle intensiv untersucht und Industrieunternehmen haben erhebliche Beträge in Pilotanlagen und Vor-Produktions-Bemühungen investiert. Bis 1985 sind alle diese Unternehmungen aufgegeben worden, und die Investitionen wurden abgeschrieben. In der Folge wurden neue industrielle Investitionen durch die aussichtsreichen Eigenschaften des amorphen Siliziums induziert, aber viele Bemühungen wurden in den Jahren nach 1990 wieder beendet. Nichtsdestoweniger zeigen Arbeiten von SOLAREX und United Solar Systems Inc. in den USA Aussichten, mit Zielkapazitäten im Bereich von $10 \mathrm{MW}$ pro Jahr spezielle Technologien zu kommerzialisieren. Eine Pilotfertigung läuft bei ASE/PST in Deutschland. Japanische Unternehmen produzieren 
kleine Silizium-Module mit etwa $8 \mathrm{MW}$ pro Jahr für die Anwendung in Konsum-Produkten, wie Rechner und Uhren. Eine kleine Fabrik wird in Frankreich von Free Energy Europe betrieben und hat eine Produktionskapazität von einigen Megawatt pro Jahr. Bemühungen um $\mathrm{CuInSe}_{2}$-basierte Solarzellen werden gegenwärtig bei Siemens durchgeführt. Im Zentrum für Solarenergie und Wasserstofftechnik (ZSW) wird eine Pilotanlage für diesen Modultyp vorbereitet. Im Falle des CdTe, das bereits 1970 als aussichtsreicher Kandidat erkannt worden ist, wurden nach 1980 mindestens drei industrielle Bemühungen zur Produktion begonnen, nämlich bei Kodak, bei AMETEK und PhotonPower/Golden Photon in den USA, aber zwischen 1985 und 1997 wieder eingestellt. Gegenwärtig laufen VorProduktionen bei Solar Cells Inc. Zwei Unternehmen planen eine großtechnische Produktion von CdTeDünnschicht-Modulen: BP-Solar in den USA und ANTEC Solar in Deutschland, beide mit einer nominellen Produktionskapazität von $10 \mathrm{MW}$ pro Jahr. Eine Produktion von kleinen CdTe-Modulen für KonsumProdukte mit einem Ausstoß von etwa einem Megawatt läuft bei Matsushita in Japan; Pläne für eine Produktion von Modulen mit CSS sind jüngst bekanntgeworden.

Aus der Analyse vergangener Fehlschläge und Erfolge können heute neue Bemühungen auf ein besseres Geschäftsfundament gestellt werden. Es ist evident, daß die meisten Abbrüche in der Pilotphase erfolgt sind, als das Management der betreffenden Unternehmen erkannt hatte, daß der Weg bis zu einer wirklichen Großproduktion länger und teurer sein würde, als die Entwicklungsleiter erwartet hatten. Die beiden wichtigsten Voraussetzungen, für den Weg zur Produktion sind:

(1) überzeugendes technisches Konzept und

(2) ein glaubhafter Geschäftsplan für eine schnelle Investition bis in die Produktion.

Heute ist es klar, daß CdTe ein ideales Material für Dünnschichtsolarzellen ist. Durch weltweite Bemühungen in Industrie und Universitäten ist ein Zustand der Technik erreicht, der die Überführung in die Produktion rechtfertigt. Wenn CdTe-Dünnschichtsolarzellen kommerziell erfolgreich werden sollen, müssen ihre vielversprechenden grundlegenden Eigenschaften in einer Art in die Produktion überführt werden, die folgende technische Kriterien erfüllt:

- hoher Wirkungsgrad (10 bis $15 \%$ ),

- hohe Produktionsgeschwindigkeit $\left(100000 \mathrm{~m}^{2}\right.$ p. a.),

- robuster, wenig sensibler Prozeß,

- billiges Substrat,

- geringer Materialverbrauch,

- Langzeitstabilität.

Art und Dauer der Investitionen haben einen starken Einfluß auf die Modulkosten der anschließenden Pro- duktion. Der Geschäftsplan sollte zeigen, daß die gesamte Investition in einem Zeitrahmen erfolgen kann, der vergleichbar mit üblichen industriellen Investitionen ist, nämlich innerhalb von drei Jahren. Wenn dies nicht erreicht werden kann, werden die Zinsen des Kapitals den zukünftigen Gewinn aufzehren. Ein finanzieller „,break-even“-Punkt sollte etwa ein Jahr nach Beginn der vollen Produktion erreichbar sein. Die folgende Diskussion zeigt, daß dies erreicht werden kann. Die einzelnen Steine des Fundaments für eine zukünftige große Produktion werden im Folgenden beschrieben und Einzelheiten der technischen und wirtschaftlichen Pläne werden identifiziert.

\section{Faktoren für eine erfolgreiche Produktion}

\subsection{Das Grundmaterial}

$\mathrm{CdTe}$ hat eine Energielücke von $1,45 \mathrm{eV}$ und ist deshalb sehr gut für eine wirksame Umwandlung des Sonnenlichts in Elektrizität geeignet. Darüber hinaus ist die Energielücke „direkt", was in einer sehr hohen Lichtabsorption resultiert, weshalb die Absorberschicht nur wenige Mikrometer dick zu sein braucht. Dies bedeutet, daß in einem Zustand der technischen Reife Wirkungsgrade von $18,5 \%$ für Solarzellen erwartet werden können, die auf CdTe basieren [3].

Bei Temperaturen über $400^{\circ} \mathrm{C}$ ist die stöchiometrische Verbindung die stabile feste Phase, da die Elemente einen erheblich höheren Dampfdruck haben, als die Verbindung. Lediglich eine geringe Nicht-Stöchiometrie führt zu einer natürlichen $\mathrm{p}$-Dotierung des Materials. Diese Eigenschaften machten es leicht, CdTeSchichten herzustellen, die für Dünnschichtsolarzellen geeignet sind.

\subsection{Der Abscheideprozeß}

Bei Erhitzung im Vakuum auf Temperaturen um $700^{\circ} \mathrm{C}$ sublimiert CdTe kongruent und setzt $\mathrm{Cd}$ und $\mathrm{Te}$ in gleichen Mengen frei, während das Residuum stöchiometrisches CdTe bleibt. Bei Ankunft von Cd und Te auf dem Substrat kondensiert stöchiometrisches CdTe, solange das Substrat sich auf Temperaturen über $450^{\circ} \mathrm{C}$ befindet. Da das Material natürlich p-dotiert aufwächst, ist keine zusätzliche Dotierung notwendig. Sauerstoff, der gleichwertig zu Cd ist, stellt keine sehr schädliche Verunreinigung dar und kann unter Umständen die Dotierung erhöhen [4].

ANTEC hat den Prozeß der Close-Spaced-Sublimation für die Schichtabscheidung gewählt (siehe Abb. 1). Ein großflächiger Graphit-Tiegel enthält das Ausgangsmaterial CdTe als Granulat und wird auf eine Temperatur von etwa $700^{\circ} \mathrm{C}$ erhitzt. Das Substrat befindet sich oder bewegt sich (in der Produktion) über dem Tiegel 
in geringem Abstand (close-spaced) und wird auf etwa $500^{\circ} \mathrm{C}$ gehalten. Bei Abscheideraten von über $10 \mu \mathrm{m}$ pro Minute wurden bei ANTEC Wirkungsgrade von etwa $12 \%$ erreicht und Module mit $(10 \cdot 10) \mathrm{cm}$ Fläche mit Wirkungsgraden in Höhe von $10,5 \%$ hergestellt.

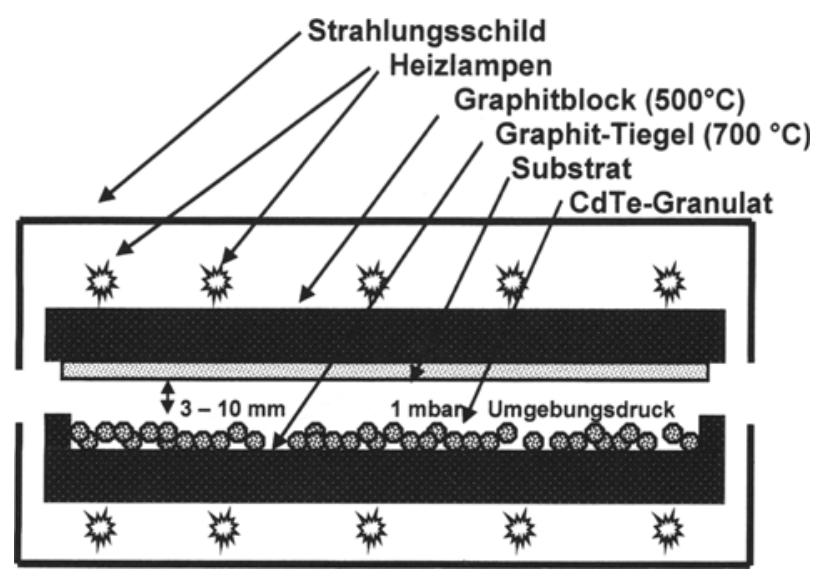

Abb. 1. Schema des CSS-Prozesses

\subsection{Die Zell- und Modul-Technik}

Wie oben erwähnt, neigt CdTe dazu, bei üblichen Herstellungsverfahren ohne weitere Dotierung p-leitend zu werden. Ein flacher p-n-Übergang (ähnlich wie in Si-Zellen) ist in CdTe-Dünnschichten schwer herzustellen und leidet überdies (wie vom GaAs bekannt) unter starker Oberflächenrekombination, was den Photostrom stark reduziert. Ein n-leitender Halbleiter ist als Partner zum Aufbau einer p-n-Diode jedoch nötig, um eine starke Raumladungszone als Voraussetzung für guten Wirkungsgrad erreichen. Ein Partner mit höherer Energielücke, als sie CdTe hat, erlaubt es Lichtquanten, das CdTe unmittelbar an der Grenzfläche zum CdTe zu erreichen. Dieser Effekt wird „window-effect" genannt. Um 1970 wurde eine neue Heterodiode für CdTe identifiziert, die CdS als n-Partner benutzt [5]. Das hat zu erheblichen Erfolgen in vielen Laboratorien geführt [6]. Wie CdTe hat CdS eine starke Neigung, stöchiometrische Filme zu bilden, aber - anders als CdTe - sind CdS-Filme von Natur aus n-dotiert. Weiterhin kann CdS mit den gleichen Abscheideverfahren wie CdTe aufgebracht werden. Ein potentieller Nachteil liegt darin, daß CdS eine gewisse Gitterfehlanpassung zum CdTe hat. Durch eine Aktivierung nach der Abscheidung kann jedoch die Grenzfläche zwischen $\mathrm{CdS}$ und $\mathrm{CdTe}$ stabilisiert bzw. verbessert werden. Diese Aktivierung wird durch Tempern der CdS/CdTe-Doppelschicht in Gegenwert von Chlorverbindungen bewirkt.

Bei der Herstellung von CdTe-Solarzellen wird zunächst eine dünne leitfähige Oxidschicht - typischerweise $\mathrm{In}_{2} \mathrm{O}_{3}$ oder $\mathrm{SnO}_{2}$ - auf Glas abgeschieden. Als nächstes werden die $\mathrm{CdS}$ - und $\mathrm{CdTe}$-Schicht abge-

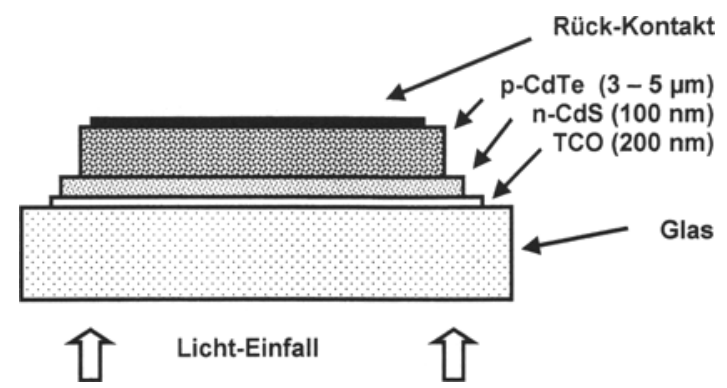

Abb. 2. Aufbau des Schichtsystems der CdTe-Dünnschichtsolarzelle

schieden und zusammen aktiviert. Schließlich wird ein niederohmiger Kontakt an die CdTe-Schicht aufgebaut, der von einer metallischen Rückelektrode gefolgt wird, die lichtundurchlässig sein kann. Abb. 2 zeigt die Superstrat-Zellstruktur, die so genannt wird, weil, wenn sie von der Sonne gesehen wird, das Glas sich „über" der eigentlichen Zelle befindet.

Bereits vor über zehn Jahren wurde anhand der amorphen Silizium-Solarzellen gezeigt, daß sich Dünnschichtsolarzellen besonders gut zur Herstellung integriert verschalteter Module eignen. Ein typisches Modul hat etwa hundert individuelle Zellen pro Meter seiner Länge, die durch die Anwendung von drei Sätzen von Trennschnitten während der Zellherstellung erzeugt werden. Schmale einzelne Zellen, die parallel zur einen Kante des Substrats laufen, werden definieret und seriell verschaltet. Die obere Elektrode von Zelle 1 berührt die untere transparente Elektrode von Zelle 2, während sie von der oberen Elektrode der Zelle 2 getrennt ist. Die obere Elektrode von Zelle 2 berührt die Grundelektrode von Zelle 3 und so fort. Abb. 3

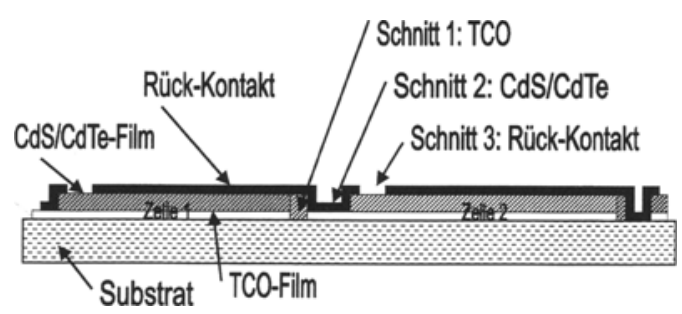

Abb. 3. Schematische Darstellung der integrierten Verschaltung von Dünnschichtmodulen

zeigt dieses Prinzip. Die Schnitte durch die einzelnen Schichten werden oft mit dem Laser ausgeführt, z. B. dem Nd:YAG-Laser. In CdTe-Dünnschichtsolarmodulen wird dies auch für die TCO-Schicht benutzt. Für die Halbleiterschicht kann eine andere Technik benutzt werden: Die CdTe-Schicht haftet sehr gut auf der transparenten Elektrode, die sehr glatt und hart ist. $\mathrm{CdTe}$ andererseits ist sehr spröde und weich, was zuläßt, daß der zweite und dritte Schnitt mit mechanischen Werkzeugen ausgeführt werden kann, die das CdTe schneiden und auf der transparenten Leiter- 
schicht gleiten, ohne sie zu schneiden. Der dritte Schnitt kann ebenso mit einem mechanischen Werkzeug erfolgen.

\subsection{Leistung von Modulen und Zellen}

Nachdem im Jahr 1992 ein Rekordwirkungsgrad von $15,8 \%$ an Zellen gemessen wurde, die mit hochwertigen Forschungs-Verfahrenschritten erzeugt worden waren [7], wurden jüngst Wirkungsgrade von $14 \%$ mit Prozessen erreicht, die für großtechnische Produktion geeignet sind [8]. Für zukünftige Zellen aus CdTe können Wirkungsgrade von 18,5\% erwartet werden [3]. Es kann wohl angenommen werden, daß unter einer reifen Produktionstechnik innerhalb von wenigen Jahren große Module mit $14 \%$ Wirkungsgrad und hoher Ausbeute produziert werden können.

Erste Langzeit-Tests im Freiland wurden von BP Solar und Solar Cells Inc. an technischen Modulen durchgeführt [9]. Sie haben nach 20000 Stunden Dauerbelichtung nur geringe Degradation gezeigt. Dies ist ein starker Hinweis darauf, daß Langzeitstabilität erreicht werden kann. Ein erheblicher Vorteil im Hinblick auf eine lange Lebensdauer liegt in der Tatsache daß anders als bei konventionellen Modulen - kein empfindliches Polymer vom Licht durchquert werden muß, bevor es den Halbleiter trifft.

\subsection{Produktionskapazität, Kosten, Leistungen}

Grundlegende wirtschaftliche Erwägungen zeigen, daß für die meisten Produktionsverfahren die Kosten für die Anlagen weniger als proportional mit der Produktionskapazität ansteigen. Eine jüngste Studie hat gezeigt, daß spezifische Kosten von 0,6 EURO pro Watt bei einer Kapazität von $60 \mathrm{MW}$ pro Jahr erreicht werden können [1]. Um die ,dimensions of scale“ bei einer nicht $\mathrm{zu}$ hohen Investition $\mathrm{zu}$ erreichen, sollte eine Kapazität von $10 \mathrm{MW}$ (etwa $100000 \mathrm{~m}^{2}$ ) pro Jahr als Ziel für eine erste Anlage gewählt werden. Diesen Überlegungen zufolge hat sich bei ANTEC Solar gezeigt, daß bei einer Kapazität von $10 \mathrm{MW}$ pro Jahr spezifische Kosten von 1 EURO pro Watt bei einer Ausbeute von $70 \%$ erreicht werden können. Bei kontinuierlichem Betrieb liegt die lineare Produktionsgeschwindigkeit einer solchen Anlage bei etwa einem Meter pro Minute. Mit dem ProzeB der Close-SpacedSublimation kann diese Kapazität leicht erreicht werden. Es wird erwartet, daß Module von etwa $9 \%$ Wirkungsgrad nach ersten Anlageoptimierungsungsschritten produziert werden können. Die Breite der Verteilungskurve ( $N$ gegen Wirkungsgrad) ist kritisch für die Ausbeute bei der nominellen Effizienz. Auf Grund der starken Unempfindlichkeit kritischer Abscheideschritte gegen Parameter-Fluktuation wird eine schmale Kurve erwartet.

\subsection{Die Investition}

Die Investitionskosten, die für dieses Unternehmen benötigt werden, wurden von professionellen Betriebswirten mit 30 Mio. EURO geschätzt. Die eigentlichen Anlagekosten wurden mit etwa $20 \mathrm{Mio}$. EURO geschätzt, konsistent mit früheren rohen Schätzungen des Autors [10] und jüngsten Ankündigungen seitens BP Solar für ihre geplante Fabrik in den USA [11]. Dieses Budget deckt alle Aktivitäten und Investitionen bis zur Aufnahme der vollen Produktion.

\section{Die Unternehmung}

\subsection{Finanzierung, Organisation, Zeitplan}

Selbst unter den grundlegenden technischen und wirtschaftlichen Vorteilen, wie sie oben illustriert wurden, kann ein solches Unternehmen fehlschlagen, wenn die Dauer des Aufbaus zu lange ist und die wirtschaftliche oder strategische Position der finanzierenden Industrieeinheit sich ändert. Die Erfüllung der zwei folgenden Aufgaben wurde als äußerst wichtig für die Planung angesehen:

(1) Sicherungen der Finanzierung von der Engineeringphase bis in die Produktion durch Errichtung einer starken und verbindlichen Partnerschaft.

(2) Durchschreiten der Investitionsphase bis zum Start der Produktion so schnell wie möglich, um Zinskosten zu minimieren (fast track).

Im Jahr 1997 wurde ANTEC Solar GmbH als Industrieunternehmen in Rudisleben (Thüringen) gegründet, das einzig und allein der Produktion von CdTeDünnschicht-Solarmodulen gewidmet ist. Gesellschafter sind zwei Personen (als Gründer) sowie ANTEC GmbH und Balke-Dür AG. Das gesamte Investitionsbudget wurde unter Einsatz von Existenzgründungsdarlehen der Personen, Investitionen des Industriepartners, Kredite von Staats- und Geschäftsbanken (garantiert durch Landes- und Bundesbürgschaften) und Subventionen für industrielle Investitionen im östlichen Teil Deutschlands (unter Zustimmung der EG) sichergestellt. Das Budget von 60 Mio. DM wird den Markteintritt mit den neuen ANTEC-Modulen ermöglichen.

ANTEC Solar tätigt die Investitionen auf die schnellste mögliche Weise wie folgt: Balke-Dürr AG handelt als Generalunternehmer und baut und liefert die Produktionsanlage schlüsselfertig innerhalb von zwei Jahren. ANTEC GmbH wird im Rahmen eines Unterauftrages von Balke-Dür AG die Engineering-Parameter in ihrer endgültigen Form erarbeiten und dafür eine kleine VorProduktions-Anlage einsetzen, die es erlaubt, Module einer Größe von $(30.60) \mathrm{cm}$ herzustellen. Die Engineering-Aktivität und der Bau der Produktionslinie wurden zeitlicher ineinander geschoben und vernetzt. Sobald eine spezielle Komponente der Produktionsli- 
nie gebaut und installiert ist, werden die Betriebsbedingungen, die unterdessen von ANTEC GmbH entwickelt worden sind, in die Anlage umgesetzt. Nach ihrer Installation wird die gesamte Anlage für eine Dauer von sechs Monaten optimiert und die volle Produktion wird zweieinhalb bis drei Jahre nach Beginn der Unternehmung starten. Die Unternehmung wurde Ende 1997 auf den Weg gebracht und in einer Pressekonferenz in Bonn am 16. März 1998 durch den Ministerpräsident des Bundeslandes Thüringen zusammen mit dem Bundesminister für Erziehung, Wissenschaft, Forschung und Technologie angekündigt. Alle Bestellungen für Anlage-Komponenten sind im Mai 1998 erfolgt.

\subsection{Die Produktionslinie von ANTEC Solar}

Die Produktionsanlage wurde als eine einzige Durchlauf-Dünnschicht-Prozessierungseinheit definiert. Der technische Kern der Abscheideprozeßlinie wird vollautomatisch mit minimalem Personaleinsatz betrieben. Nur in dem Modul-Montageanlagenteil (Laminierung, Kontaktierung, Vermessung) werden Komponenten von Hand bedient werden. Eine Datenverarbeitung und Aufzeichnung wird es erlauben, jedes Modul durch die Linie zu verfolgen.

In Rudisleben nahe bei Erfurt im Land Thüringen wurden zwei existierende Produktionshallen mit je $3000 \mathrm{~m}^{2}$ Grundfläche gemietet. Sie werden gegenwärtig für die Produktionsanlage renoviert. Die Linie mit einer gesamten Länge von $180 \mathrm{~m}$ wird einmal gefaltet und am Ende der Halbleiter-Abscheidungskomponenten abgeschnitten und in Halle 2 fortgesetzt. Halle 1 und 2 sind durch einen Korridor verbunden. Reine Bedingungen aber keine eigentlichen Reinraumbedingungen sind für die Anlage nötig. Nur in den ersten Prozessschritten wird das Substrat durch LaminarFlow-Boxen geschützt. Anlieferung von Glas und Versand der Module (etwa 2000 t pro Jahr) auf der Straße wird durch einen neuen Autobahnanschluß erleichtert, der im Bau ist.

Die Installation der Anlage wird zu Beginn des Jahres 1999 stattfinden, nachdem die notwendige Infrastruktur vorhanden ist. Abb. 4 zeigt die Anordnung der Komponenten der Produktionslinie. Mit einer Taktzeit von $90 \mathrm{~s}$ werden Module einer Größe von $(60-120) \mathrm{cm}$ gefertigt. Alle Anlagenkomponenten sind dieser Geschwindigkeit angepaßt. ANTEC hat die grundlegenden Spezifikationen aller Komponenten für die zehn Megawatt-Produktionsanlage fertiggestellt. Auf dieser Basis wurden die Anlagen von Balke-Dürr AG bestellt. Die Feinabstimmung der Prozesse und das Tuning wird, wie erwähnt, bei ANTEC GmbH anhand einer Pilotanlage durchgeführt. Die Resultate werden in die Produktionsanlage übertragen. Im folgenden werden die einzelnen Schritte der Modul-Produktion (Glas zum Modul) kurz beschrieben:

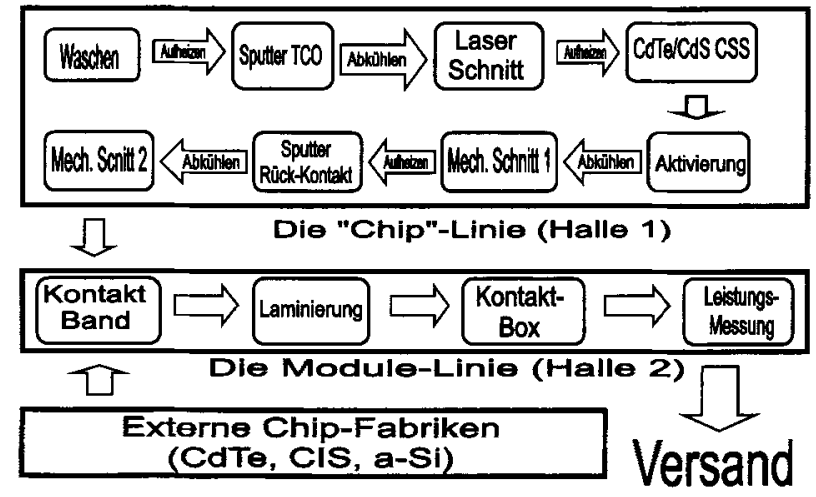

Abb. 4. Die Produktionslinie in Einzelschritten

\subsubsection{Reinigung}

Kommerzielles Fensterglas wird auf Maß geschnitten in die Fabrik geliefert, ausgepackt und in die erste Einheit der Produktionslinie, eine kommerzielle GlasWaschanlage, eingebracht.

\subsubsection{TCO-Abscheidung}

Es wurde die Entscheidung getroffen, kein fertig TCObeschichtetes Glas zu kaufen, sondern die Abscheidung in der Linie direkt durchzuführen, aus zwei Gründen:

(1) gegenwärtige Lieferquellen für TCO-beschichtetes Glas sind nicht zuverlässig, zweite Quellen (second source) sind nicht verfügbar,

(2) reinigen von TCO-beschichtetem Glas ist schwieriger als von Fensterglas.

Die TCO-Schichten werden in-line auf bewegtes Glas durch Kathodenzerstäubung mit kommerziellen Routineprozessen aufgebracht. $\mathrm{In}_{2} \mathrm{O}_{3}$ und/oder $\mathrm{SnO}_{2}$ werden benutzt, eine Flächenleitfähigkeit von etwa $7 \Omega / \mathrm{cm}^{2}$ und eine optische Transmission von $>80 \%$ sind erforderlich. Die Schichtdicken werden bei fester Durchlaufgeschwindigkeit außer durch die Prozeßparameter durch die Anzahl der Quellen für jede Schicht festgelegt.

\subsubsection{Trennschnitt 1}

Dieser Schritt bestimmt die Breite er einzelnen Zellen, die in Serie verschaltet werden. Für das Material CdTe und den gegebenen Flächenwiderstand der TCOSchicht liegt die optimale Zellbreite bei $8,5 \mathrm{~cm}$. Die TCO-Schicht wird in periodischen Linien einer Breite von etwa $100 \mu \mathrm{m}$ durch Laserablation entfernt. Dies ist ein Standardprozeß, für den es bereits kommerzielle Verfahren gibt. Für ein Modul mit $120 \mathrm{~cm}$ Länge werden 138 Zellen in Serie verschaltet.

\subsubsection{CdS-Abscheidung}

Nach Trennschnitt Nr. 1 bewegen sich die Substrate durch eine Schleuse in eine Vakuumanlage und werden auf die Abscheidungstemperatur von $500^{\circ} \mathrm{C}$ erhitzt. Sie bewegen sich anschließend durch die CSS-Abscheide- 
kammer in geringem Abstand über der CdS-Sublimations-Quelle. Eine dünne etwa $100 \mathrm{~nm}$ dicke, n-leitende CdS-Schicht wird abgeschieden. Durch Anpassen der Quelltemperatur wird die Abscheiderate so festgelegt, daß sie zur Laufgeschwindigkeit der Substrate konform ist. Auf Grund der geringen Anforderungen an die Qualität des Vakuums werden nur mechanische Pumpen benötigt, die zu einer erheblichen Reduktion der Anlagekosten und Wartungsanforderungen führen.

\subsubsection{CdTe-Abscheidung}

Ohne Luftkontakt bewegen sich die Substrate unmittelbar weiter in die CdTe-Abscheidekammer und werden ebenfalls durch Close-Spaced-Sublimation mit einer $3 \mu \mathrm{m}$ bis $7 \mu \mathrm{m}$ dicken CdTe-Schicht bei der gleichen Substrattemperatur beschichtet. Irgendwelche Fluktuationen in der Schichtdicke des CdTe sind nicht kritisch, da nur das erste Mikrometer der CdTe-Schicht zum Photostrom beiträgt. Für beide Prozesse müssen nur zwei Parameter eingestellt werden, die Temperatur der Quelle und des Substrats.

\subsubsection{Aktivierung}

An dieser Stelle bewegt sich das Substrate in die Aktivierungskammer, wo es in Gegenwart eines Cl-haltigen Gases getempert wird. Dies findet bei etwas niedrigerer Temperatur statt.

\subsubsection{Trennschnitt 2}

Nachdem das Substrat eine Abkühlungsstufe durchlaufen hat, tritt es aus der Anlage aus und bewegt sich in die erste Einheit zur mechanischen Trennung. Ein einfaches oder vielfaches Werkzeug erodiert Sätze von bis zu 20 Linien in die CdTe-Schicht parallel zu und sehr dicht an den ersten Laser-ablatierten Linien in dem TCO.

\subsubsection{Abscheidung des Rück-Kontakts}

Der Rück-Kontakt besteht aus einer Doppelschicht, einer Halbleiterschicht und einer Metallschicht. Beide werden durch Kathodenzerstäubung in einem Durchlaufsystem auf bewegte Substrate abgeschieden.

\subsubsection{Trennschnitt 3}

Dieser Schritt trennt den Rückkontakt der individuellen Zellen durch mechanische Bearbeitung. Das Werkzeug kann bis zur TCO-Schicht hinunter schneiden, so daß der Prozeß identisch dem zweiten Schnitt ist. An dieser Stelle werden Module ,Chip-Module“ genannt, um ihre noch unverkapselte Situation zu charakterisieren.

\subsubsection{Isolation, Kontaktierung, Laminierung}

Ein Streifen von etwa $0,5 \mathrm{~cm}$ Breite am Glasrand wird von leitfähigem Material durch mechanische Ablation befreit, um dem Modul den erforderlichen Hochspannungsschutz zu geben. Metallbänder wer-

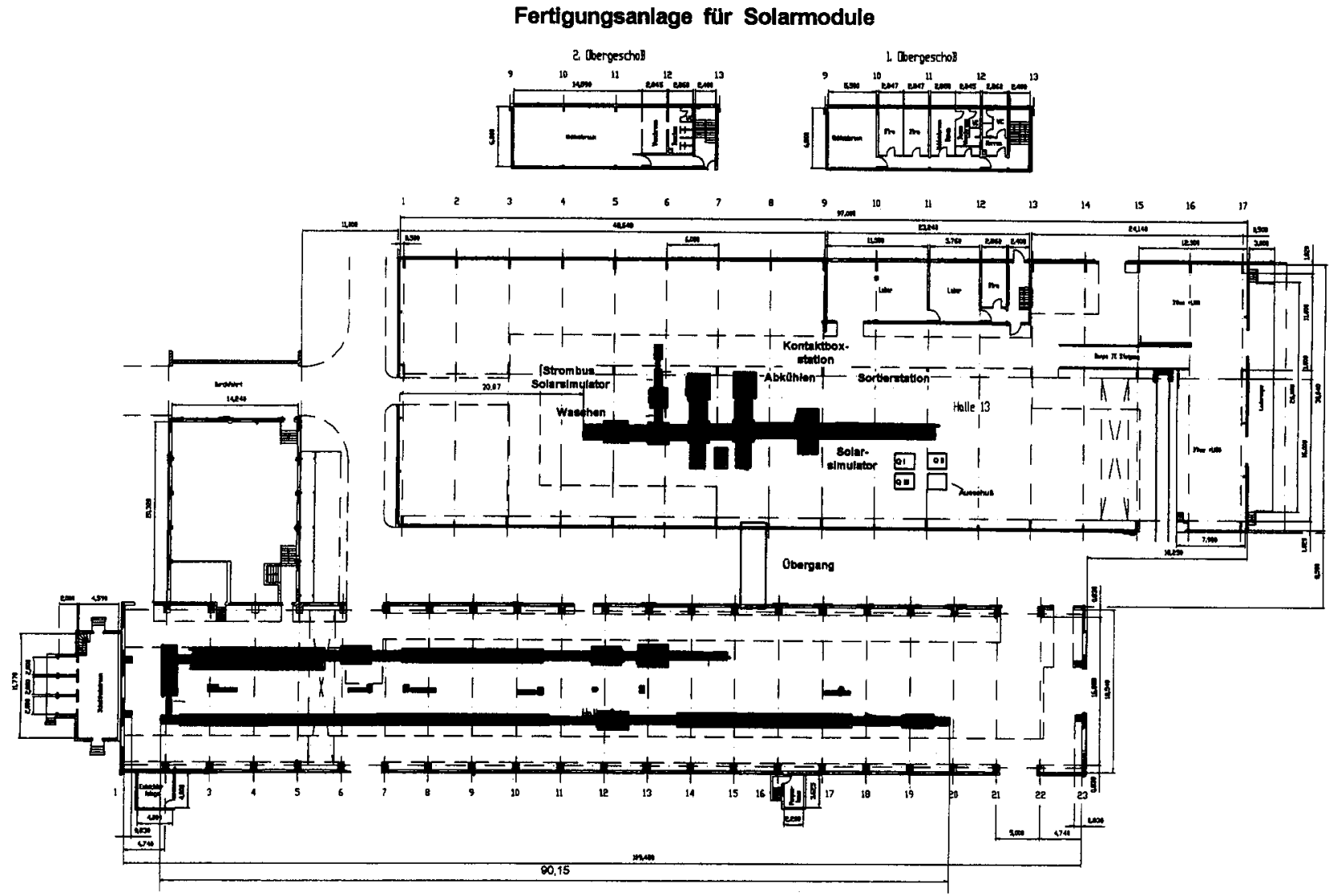

Abb. 5. Grundriß der Fabrik 


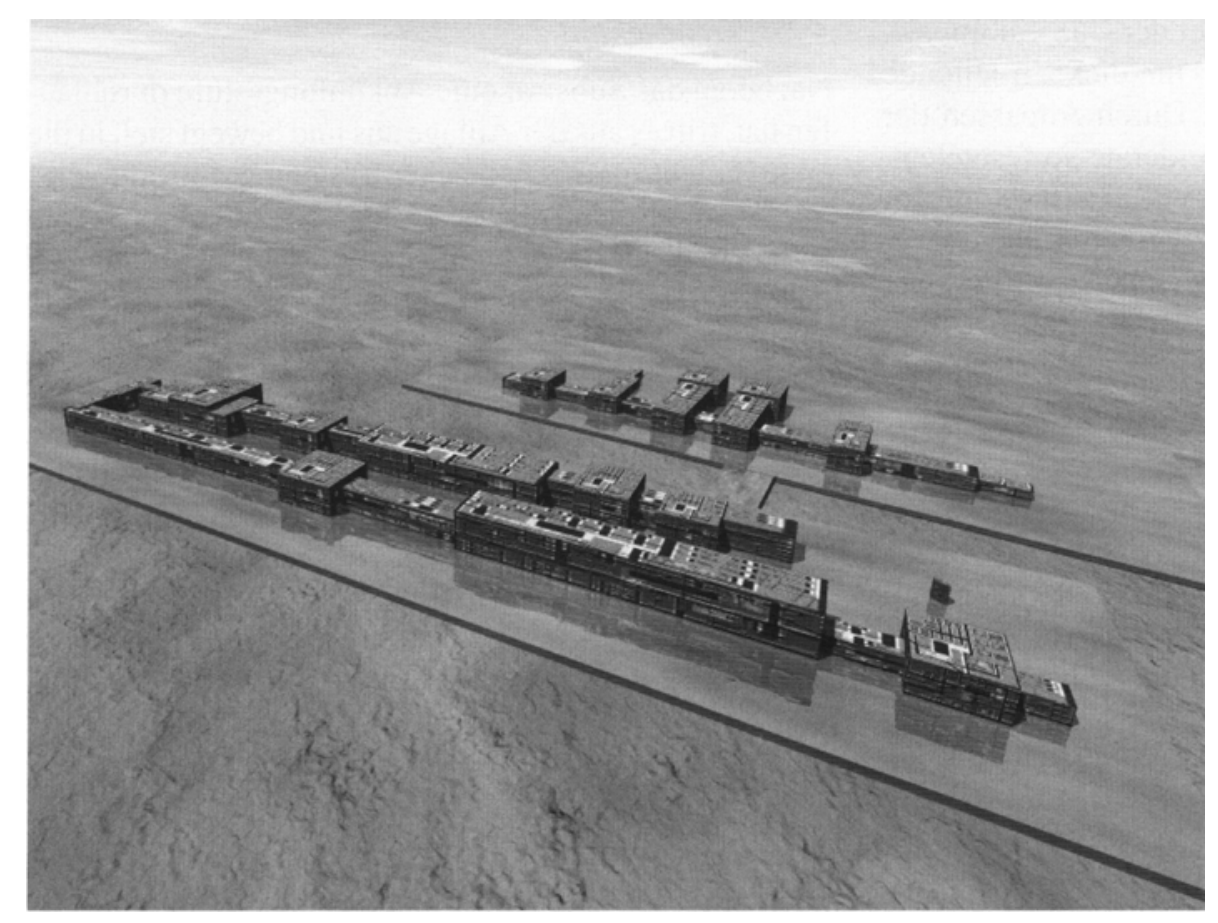

Abb. 6. Artist's View der Anlage

den an die Kontakte der äußersten Zellen angebracht und durch geeignete Löcher im Rückglas herausgeführt. Dieses Glas wird auf das Modul-Glas, das Chip-Glas, durch Standardprozesse laminiert. Eine spezielle EVA-Folie wurde ausgewählt und die Laminierungstechnik wird optimiert. Kontaktboxen werden an dieser Stelle, entsprechend der gewünschten Anwendung, angebracht.

\subsubsection{Leistungsmessung, Inspektion, Qualifizierung}

Jedes Modul wird unter einem Blitz-Solarsimulator gemessen und die Daten werden gespeichert. Module mit Unterleistung werden ausgeschieden. Module mit ausreichender Leistung werden etikettiert, inspiziert, verpackt und dem Versand zugeführt.

\subsection{Risikobewertung}

Vor dem Start des Unternehmens wurde eine umfassende Risikobewertung durchgeführt und zwischen den Ingenieuren und den finanzierenden Gruppen abgestimmt. Das zugeordnete Budget wurde als ausreichend angesehen, um alle Risiken abzufangen.

\section{Einfahren der Fabrik}

Um die Fabrik möglichst schnell zu aktivieren und hochzufahren sowie um den Markt so schnell wie möglich zu öffnen, wird der Betrieb in zwei Phasen aufgenommen werden: In Phase 1 wird lediglich die Modul-Linie in Halle 2 betrieben. Ungekapselte Module, Chips, werden durch Geschäftspartner gelie- fert und werden laminiert und entsprechend der vorgesehenen Anwendung konfektioniert. Phase 1 wird im vierten Quartal 1998 operativ werden. In Phase 2 wird Halle 1 mit der Chip-Linie eingefahren und an Halle 2 angekoppelt. Volle Kapazität wird im Jahre 2000 erreicht. Abb. 5 zeigt den Grundriß der Fabrik, Abb. 6. gibt einen artist's view der zukünftigen Anlage.

\section{Schluß}

Das Ergebnis einer Übersicht, die im Jahre 1995 über CdTe als Material für Solarzellen geschrieben wurde, findet sich die folgende Formulierung von Peter Meyers und William Birkmire [12]:

„The real strength of CdTe PV technology, however, is in its suitability for high-throughput manufacturing and low manufacturing cost."

Dies wurde durch ANTECs-Analysen und experimentelle Arbeiten bestätigt. Die Zeit ist reif, um in die Großproduktion so schnell wie möglich einzutreten.

\section{Schrifttum}

[1] Woodcock, J. M., Schade, H., Maurus, H., Dimmler, B., Springer, J., Ricaud, H.: Proc. 14th European Photovoltaic Solar Energy Conf. 1997, S. 857 bis 860.

[2] Bruton, T. M., Luthardt, G., Rasch, K.-D., Roy, K., Dotty, I. A., Garrard, B., Teale, L., Alonso, J., Ugalde, U., Declerq, K., Nijs, J., Szlufciuk, J., Räuber, A., Wettling, W., Vallêra, A.: Proc. 14th European Photovoltaic Solar Energy Conf. 1997, S. 11 bis 16. 
[3] Sites, J. R., Lui, X.: Progress in Photovoltaics 3 (1995), S. 307 bis 314 .

[4] Tyan, Y.-S., Perez-Albuerne, E. A.: Conf. Record of the 16th Photovoltaic Specialists Conference 1982, S. 794 bis 800 .

[5] Bonnet, D., Rabenhorst, H.: Conf. Record of the 9th Photovoltaic Specialists Conf. 1972, S. 129 bis 131.

[6] Bonnet, D.: Proc. 14th European Photovoltaic Solar Energy Conf. 1997, S. 2688 bis 2693.

[7] Chu, T. L., Chu, Sh., Britt, J., Chen, G., Ferekides, C., Ullal, H. S.: Proc. 11th European Photovoltaic Solar Energy Conf. 1992, S. 988 bis 990.
[8] Ferekides, C., Marinski, D., Morel, D. L.: Conf. Record of the 26th Photovoltaic Specialists Conf. 1997, S. 339 bis 342 .

[9] Sasala, R. A., Powell, R. C., Dorer, D. L., Reiter, N.: NREL/SNL Photovoltaics Program Review Meeting, Nov. 18-22, Lakewood CO, USA, APS Conference Proceedings 394 (1996), S. 171 bis 188.

[10] Bonnet, D.: Int. J. Solar Energy 12 (1992), S. 1 bis 14.

[11] Marshall, J.: San Francisco Chronicle, 20. 5. 1997.

[12] -Meyers, P. V., Birkmire, W.: Progress in Photovoltaics. Research and Applications 3 (1995), 393 bis 402.

\section{Zukunftschancen der Photovoltaik}

\section{F. Vahrenholt ${ }^{1}$}

Eine Darstellung der Gründe, weshalb Shell die Photovoltaik zu einem ihrer Kerngeschäfte erklärte:

- Die Endlichkeit von Öl- und Gasressourcen wird im Verlauf des nächsten Jahrhunderts spürbar.

- Bevor Knappheiten zu erheblichen Verteuerungen führen, werden die durch $\mathrm{CO}_{2}$ und Methan ausgelösten Klimaveränderungen zu einer Forderung nach Drosselung der Verbrennung fossiler Brennstoffe führen.

- Regenerative Energien haben weltweit eine große Marktchance.

Schlüsselwörter: nachhaltiges Wachstum; Dematerialisierung; erneuerbare Energien; Photovoltaik

Future chances of photovoltaics. A presentation of reasons for Shell do declare photovoltaics to once of their main trades:

- The limitation of oil and gas resources will be notable in the course of the next century.

- Before the shortnesses provoke massive increases in costs, the changes in climate caused by $\mathrm{CO}_{2}$ and methane will lead to a demand of tapering off the burning of fossile fuels.

- Regenerative energies have globally a great market chance.

Keywords: lasting growth; dematerialization; renewable energies; photovoltaics

Die Jahre 1997 und 1998 werden vielleicht einmal als die Jahre des Beginns einer Erfolgsgeschichte einer neuen Energietechnologie, der Photovoltaik, angesehen werden. Seit Jahrzehnten ist diese Technologie bekannt, aber über ein Nischen- und Mauerblümchendasein kam sie nicht hinaus.

Drei Ereignisse markieren die Trendwenden:

\footnotetext{
${ }^{1}$ Dr. Fritz Vahrenholt, Vorstandsmitglied der Deutschen Shell AG, Überseering 35, D-22297 Hamburg.
}

(1) Kyoto als Startpunkt einer globalen Zielsetzung der Entwicklung regenerativer Energien,

(2) das Weißbuch der EU mit dem ehrgeizigen Ziel bis $20103000 \mathrm{MW}$ installierte Leistung allein in Europa,

(3) und nicht zuletzt der Weltkongreß der Photovoltaik, der gezeigt hat, Photovoltaik wird ein ,core business" großer mutlinationaler Unternehmen von Siemens über Kyocerca, BP und Shell. 